\title{
Nuclear translocation of granzyme B in target cell apoptosis
}

\author{
MJ Pinkoski ${ }^{1,2}$ JA Heibein ${ }^{1}$, M Barry ${ }^{1}$ and RC Bleackley ${ }^{\star, 1}$ \\ ${ }^{1}$ Department of Biochemistry, University of Alberta, Edmonton, Alberta, \\ Canada, T6G 2H7 \\ 2 Division of Cellular Immunology, La Jolla Institute for Allergy and \\ Immunology, 10355 Science Center Drive, San Diego, California, CA, USA \\ * Corresponding author: RC Bleackley, Department of Biochemistry, University \\ of Alberta, Edmonton, Alberta, Canada, T6G 2H7. Tel: (780) 492-3968; \\ Fax: (780) 492-0886; E-mail: chris.bleackley@ualberta.ca
}

Received 16.6.99; revised 28.9.99; accepted 4.10.99

Edited by $\mathrm{C}$ Thiele

\begin{abstract}
Granzyme B is the prototypic member of a family of serine proteases localized to the cytolytic granules of cytotoxic lymphocytes. Together with another granule protein, perforin, granzyme B is capable of inducing all aspects of apoptotic death in target cells. A number of granzyme $B$ substrates have been identified and it has been demonstrated that granzyme $B$ is responsible, directly or indirectly, for the morphological nuclear changes observed in target cell apoptosis, including DNA fragmentation. In an earlier study, we showed that granzyme $B$ binds to a nuclear protein in a manner dependent on its enzymatic activity. Here, we demonstrate that granzyme B is translocated rapidly to the nucleus in cells that have been induced to undergo apoptosis by a granzymedependent process, and that translocation is dependent on caspase activity. Appearance of granzyme B in the nucleus of target cells precedes the detection of DNA fragmentation. Although not directly responsible for DNA fragmentation, these data suggest a nuclear role for granzyme $B$ in target cell apoptosis. c-Abl nuclear functions. Cell Death and Differentiation (2000) 7, 17-24.
\end{abstract}

Keywords: apoptosis; caspases; granzyme B; nuclear translocation

Abbreviations: Ad2, Adenovirus type 2; CAD, caspase activated deoxyribonuclease; CTL, cytotoxic T lymphocyte; DAPI, 4,6diamidino-2-phenylindole dihydrochloride; $\mathrm{DCl}, 3,4$-dichloroisocoumarin; $\mathrm{DiOC}_{6}(3)$, 3,5'-dihexyloxacarbocyanine iodide; DFF, DNA fragmentation factor; FITC, fluorescein isothiocyanate; ICAD, inhibitor of caspase activated deoxyribonuclease; PARP, poly (ADP-ribose) polymerase; TUNEL, terminal deoxynucleotidyl transferase-mediated dUTP-FITC nick end labeling; $\Delta \Psi_{\mathrm{m}}$, mitochondrial inner membrane potential

\section{Introduction}

Apoptosis of target cells induced by cytotoxic T lymphocytes (CTL) includes complete dismantling of the target by initiation of the apoptotic process at numerous stages. One of the key apoptotic proteins is granzyme B which, with perforin, is contained in the cytolytic granule of the CTL. Upon engagement of the target cell, the CTL secretes the contents of its cytolyic granules into the microenvironment of the target cell. ${ }^{1-4}$ New reports have shown that granzyme B enters the target cell by receptor mediated endocytosis independently of perforin, however, perforin is required to initiate granzyme Bmediated apoptosis. ${ }^{5,6}$ Granzyme B is then brought into contact with cytoplasmic substrates, notably caspase-3, as part of the activation of the apoptotic program. In addition to cytoplasmic apoptotic events involving caspase activation, there is a growing body of evidence suggesting a role for granzyme B in nuclear apoptosis. ${ }^{7-10}$

Disintegration of the nucleus is an integral step during apoptosis. ${ }^{11}$ Nuclear condensation and chromatin margination are events that were observed early in the study of apoptosis. These morphological observations were followed by the biochemical description of nuclear breakdown, which involves nucleolytic breakdown of DNA into multimers of internucleosomal sized fragments. ${ }^{12,13}$ This 'laddering' effect, first described by Duke et al. ${ }^{14}$ has become one of the signature features of apoptosis. Nuclear condensation, another hallmark morphologic feature of apoptosis, appears to occur through proteolytic breakdown of the nuclear lamina. Lamins A, B and C provide the framework on which the nuclear envelope is supported. ${ }^{15}$ Lazebnik et al. ${ }^{16}$ and Takahashi et al. ${ }^{17}$ have shown that cleavage of lamin $A$ can be attributed to caspase-6.

Granzyme B is known to participate indirectly in nuclear apoptotic events, such as DNA fragmentation, through the activation of caspase-3. Active caspase-3 subsequently cleaves the inhibitor of caspase activated deoxyribonuclease $\left(\mathrm{ICAD}^{18}\right.$ or $\mathrm{DFF}^{19}$ ) leading to the activation of the nuclease, caspase-activated deoxyribonuclease (CAD), ${ }^{20}$ as well as nuclear condensation through the activation of caspase- $6 .{ }^{16,17}$ There is a growing body of evidence suggesting a direct nuclear activity for granzyme $B^{17,21,22}$ and that nuclear uptake of granzyme $B$ into isolated nuclei is energy independent, but requires the presence of a cytosolic factor(s). ${ }^{23}$ Granzyme B binds to a nucleolar protein associated with heterochromatin in situ, ${ }^{7}$ and we now show that granzyme B undergoes rapid nuclear translocation in target cells prior to the onset of DNA fragmentation. However, accumulation of granzyme $B$ in the nucleus is drastically reduced when caspase-3-like caspases are inactivated. These studies outline the contribution of granzyme B to such nuclear events as DNA fragmentation, nuclear condensation and lamin proteolysis and demonstrate that although not directly required for DNA fragmentation, there was significant accumulation of nuclear granzyme B prior to the onset of DNA fragmentation. We also demonstrate the catalytic requirements of granzyme $B$ for its nuclear translocation. These results provide evidence to support the notion that, in addition to the cytoplasmic 

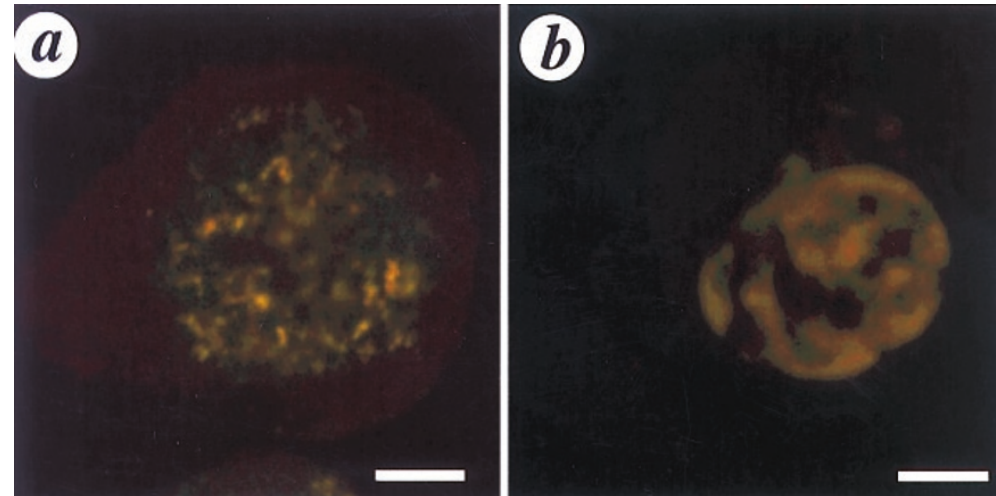

Figure 1 Nuclear translocation of granzyme B is associated with DNA fragmentation. Jurkat targets were incubated for 60 min to allow uptake of granzyme B. After washing, cells were treated with perforin (a) and Ad2 (b). Cells in all panels were labeled with anti-granzyme B antiserum and Texas Red-conjugated goat antirabbit antibody (red) and by TUNEL with dUTP-FITC (green). (A) Shows a cell in the early stages of DNA fragmentation prior to nuclear condensation shown in (B).

Regions of co-localization appear yellow. Scale bar is $7.5 \mu \mathrm{m}$

A

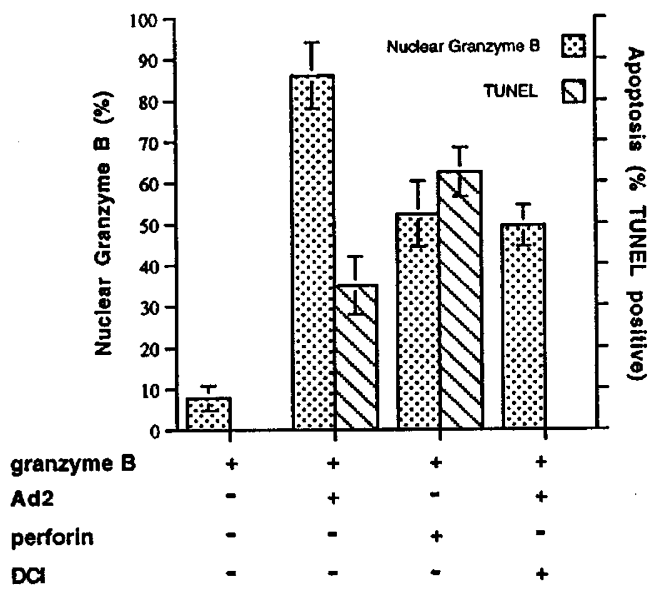

C

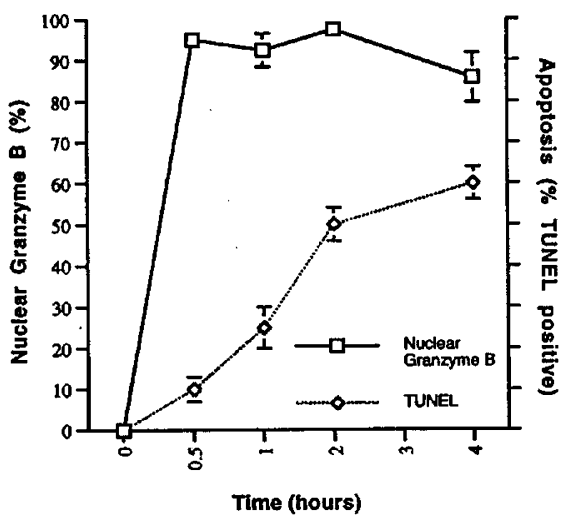

B

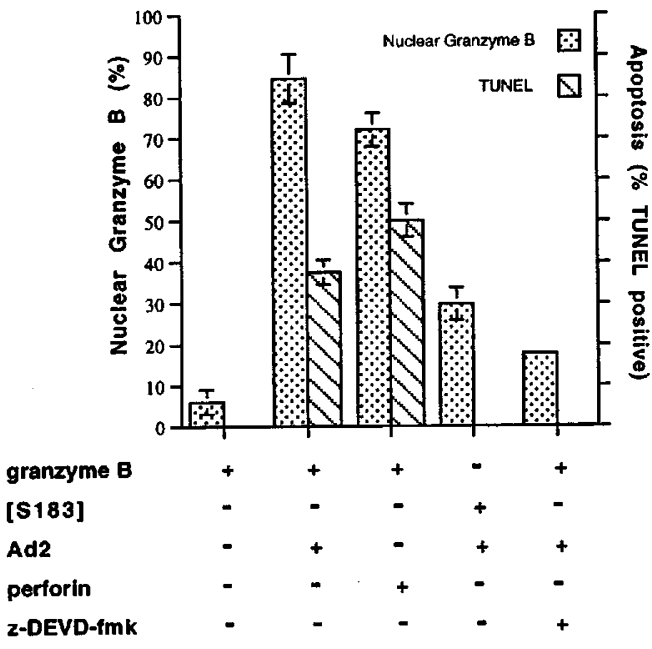

D

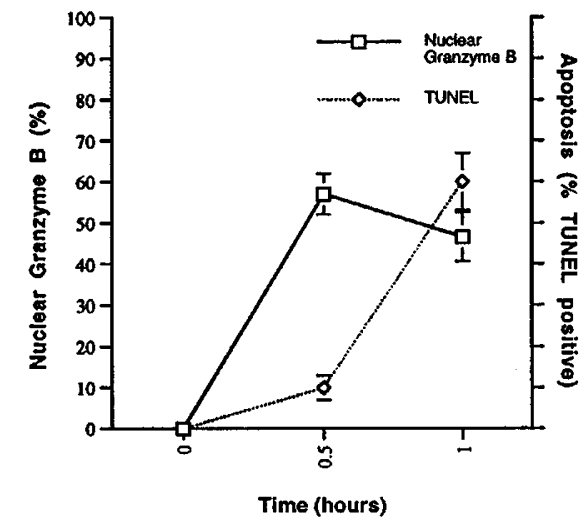

Figure 2 Quantification of nuclear granzyme B. (A) Granzyme B was added to the media of Jurkat targets for 60 min followed by addition of perforin or Ad2. In all panels, nuclear granzyme B was quantified as described in Materials and Methods and apoptosis was assayed using fluorescent TUNEL in situ. (B) COS M5 cells were transiently transfected to express granzyme B or the active site mutant, granzyme B[S183A]. At $48 \mathrm{~h}, \mathrm{Ad} 2$ or perforin was added for 60 min. In one treatment $z-$ DEVD-fmk was added for $30 \mathrm{~min}$ prior to the addition of perforin or Ad2. (C) and (D) Time course of the appearance of nuclear granzyme B and apoptosis in Jurkat target cells treated with granzyme and $\mathrm{Ad} 2$ (C) or perforin (D). Each data point represents the average of triplicate samples from at least three experiments 

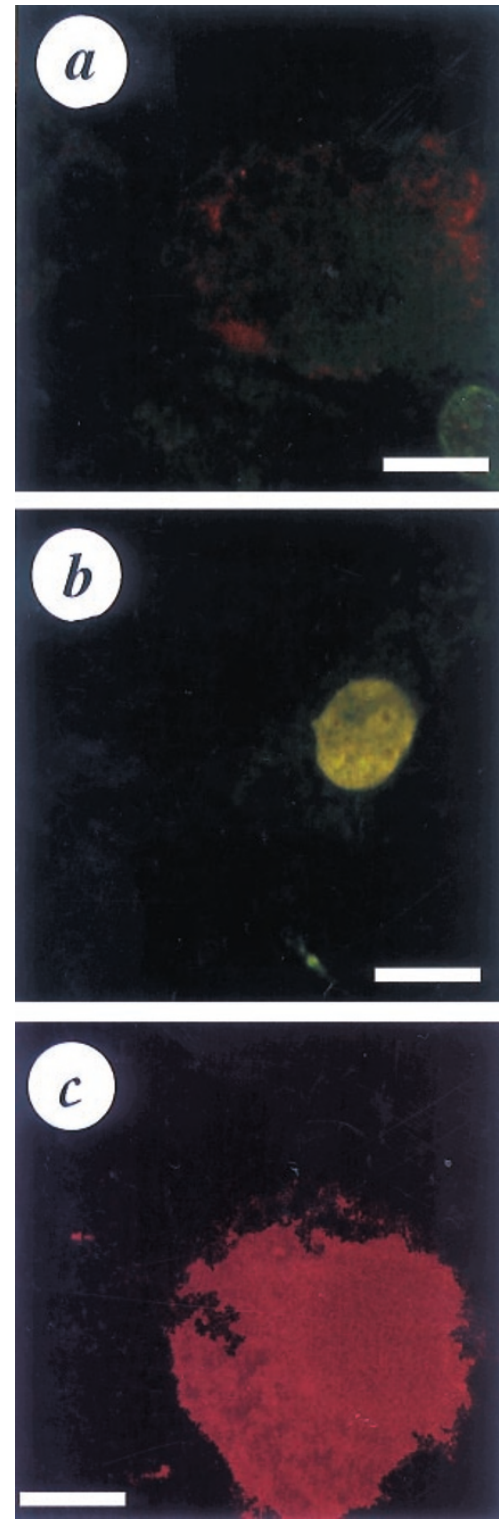

Figure 3 Apoptosis, but not nuclear translocation of granzyme, of COS M5 expressing granzyme $B$ is dependent on the presence of an active enzyme. COS M5 were transfected with granzyme $B(\mathbf{A}$ and $\mathbf{B})$, or granzyme $B[S 183 A]$ (C) followed by buffer (A) Ad2 (B and C). Cells in all panels were labeled with anti-granzyme $B$ antiserum and Texas Red-conjugated goat anti-rabbit antibody (red) and by TUNEL with dUTP-FITC (green). Regions of colocalization appear yellow. Scale bar is $10 \mu \mathrm{m}$

activities of granzyme B and its indirect association with nuclear breakdown, granzyme B may also play a direct role in nuclear apoptosis of target cells.

\section{Results}

\section{Cell death is concomitant with nuclear translocation of granzyme B}

It has been proposed that granzyme $\mathrm{B}$, in addition to cleaving cytosolic substrates, contributes to apoptosis through a mechanism that involves entering the nucleus. ${ }^{7-9}$ Therefore, we asked if, after exposure to granzyme $B$, treatment with perforin or type 2 adenovirus (Ad2) resulted in translocation of granzyme $B$ to the nucleus. Jurkat and YAC-1 target cells were incubated with granzyme $B$ and then exposed to sublytic doses of perforin or $\mathrm{Ad} 2$. Granzyme B was detected by immunolabeling and apoptosis assessed either by fluorescence TUNEL or DAPI stain and viewed by confocal laser scanning microscopy (CLSM) or fluorescence microscopy, respectively. Typical TUNEL positive Jurkat cells following treatment with granzyme $B$ and perforin or Ad2 are shown in Figure 1A,B. Granzyme B was no longer observed in the distinct punctate vesicular pattern described in an earlier report; ${ }^{5}$ rather, significant nuclear labeling of granzyme B was observed associated with regions of TUNEL label.

In order to quantify the accumulation of nuclear granzyme B, the NIH-Image software was used to analyze digital confocal micrographs to determine nuclear levels of granzyme B. Black and white micrographs were sharpened, edged and analyzed by counting grains of positive pixels with signals above a pre-set threshold. Jurkat cells pretreated with granzyme $B$ had less than $10 \%$ of the total granzyme $B$ label in the nucleus after $2 \mathrm{~h}$ (Figure 2A). This was considered to represent background for quantification by this method since it is comparable to the level of nuclear granzyme B observed in COS cells transiently transfected to express granzyme B (Figure 2B). After treatment with perforin, up to $57 \%$ of the total granzyme B was nuclear. Ad2 infection of granzyme B treated cells resulted in even greater levels of nuclear granzyme ( $>80 \%$ nuclear). Interestingly, detection of granzyme B in the nuclei of target cells preceded DNA fragmentation and cell death (Figure 2C).

\section{Enzymatic activity of granzyme $B$ is required for apoptosis and for efficient entry into the target cell nucleus}

We next asked if catalytically active granzyme B was necessary for nuclear translocation and DNA fragmentation. In the first approach, the cellular distribution of inactive granzyme was determined. Granzyme B was chemically inactivated with the serine protease inhibitor, 3,4-dichloroisocoumarin (DCl), which is known to inhibit granzyme B activity. ${ }^{24} \mathrm{DCl}$-inactivated granzyme B was then added to target cells in the presence and absence of Ad2. Cells were immunolabeled for detection of granzyme B and analyzed by CLSM. The distribution of granzyme B in targets treated with the inactive protease alone was limited to the cytoplasm. However, following treatment with Ad2, the inactive granzyme underwent translocation to the nucleus (Figure 2A). Prior inactivation of granzyme $\mathrm{B}$ with $\mathrm{DCl}$ reduced the amount of nuclear granzyme signal, but still showed nearly half of the total granzyme B localized in the nucleus within 60 min following Ad2. Importantly, the target cells did not display evidence of DNA fragmentation by terminal deoxynucleotidyl transferase-mediated dUTP nick end lableing (TUNEL) or phosphatidylserine externalization from the inner to the 

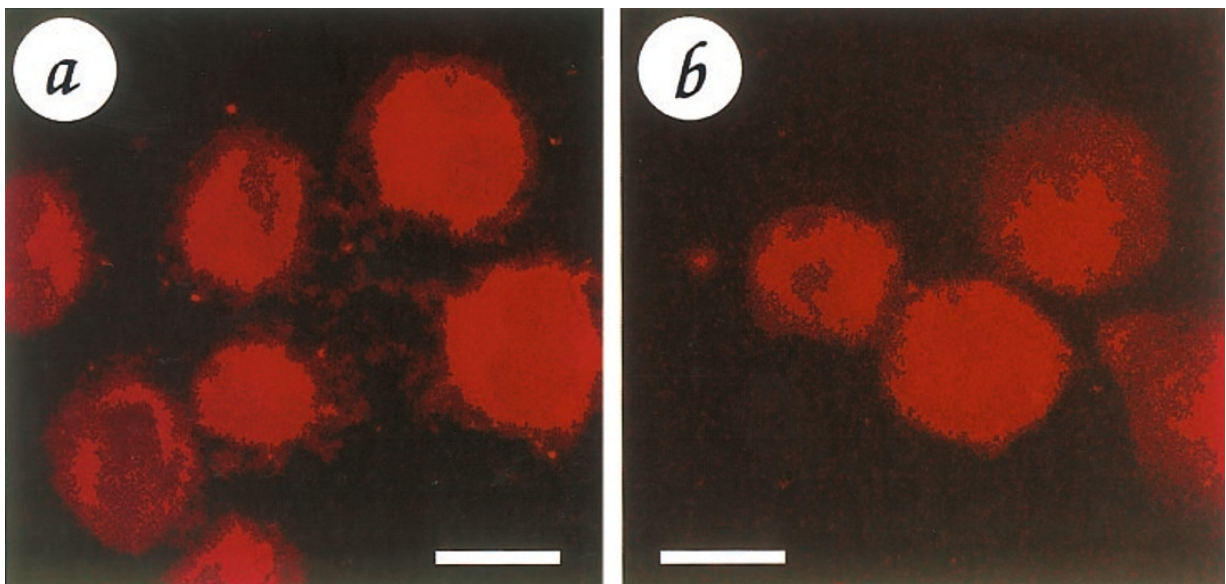

Figure 4 Inhibition of caspase-3 inhibits DNA fragmentation but not nuclear translocation of granzyme B. Jurkat targets were treated with $z$-DEVD-fmk for 30 min prior to incubation with granzyme B for $60 \mathrm{~min}$ and perforin $(\mathbf{A})$ or $\mathrm{Ad2}(\mathbf{B})$ for an additional $60 \mathrm{~min}$ (all at $37^{\circ} \mathrm{C}$ ). Cells were labeled with $\alpha$-granzyme $B$ and Texas Red $^{\mathrm{TM}}$-conjugated secondary antibody and TUNEL with dUTP-FITC. A lack of green label in these cells is indicative of the absence of DNA fragmentation. However, there is a significant nuclear accumulation of granzyme B. Scale bar is $10 \mu \mathrm{m}$
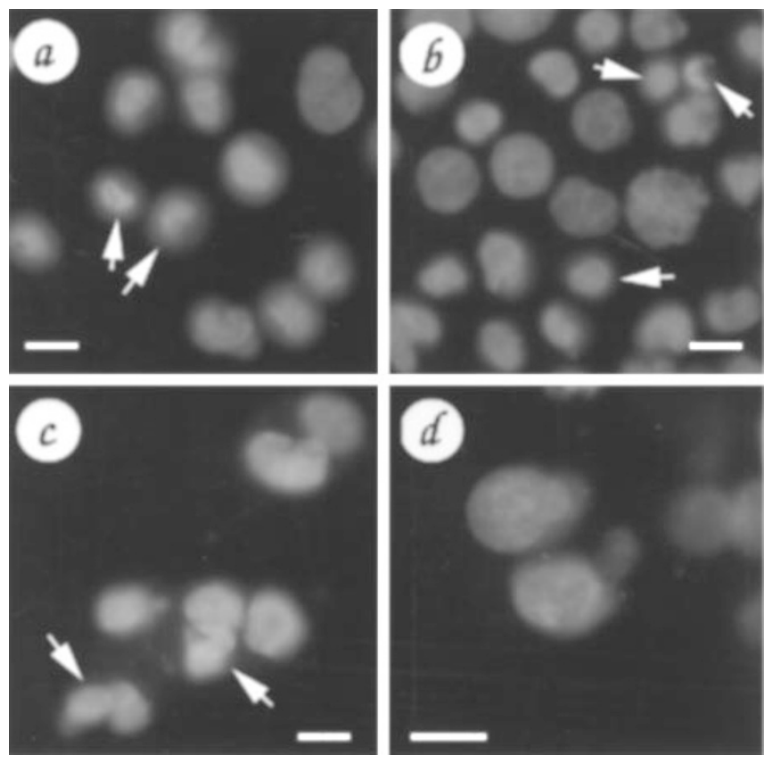

Figure 5 Nuclear condensation occurs in the absence of DNA fragmentation: inhibition of caspase-3 inhibits DNA fragmentation but not nuclear condensation. (A) and (B) show Jurkats that were incubated for $60 \mathrm{~min}$ to allow uptake of granzyme B. Cells were washed and incubated with the caspase-3 inhibitor, $z$-DEVD-fmk, followed by treatment with sublytic levels of perforin (A) and Ad2 (B). Cells were stained with DAPI for nuclear morphology. COS M5 expressing granzyme $B$ and granzyme B[S183A] treated with sublytic levels of perforin, stained with DAPI, are shown in (C) and (D) respectively. Arrows highlight examples of condensed nuclei in apoptotic cells. Scale bar is $10 \mu \mathrm{m}$

outer leaflet of the plasma membrane as detected by annexin V-FITC binding (data not shown).

In a second approach, trafficking of granzyme B was evaluated in COS M5 cells that expressed either active proteinase or the active site mutant, granzyme B[S183A].

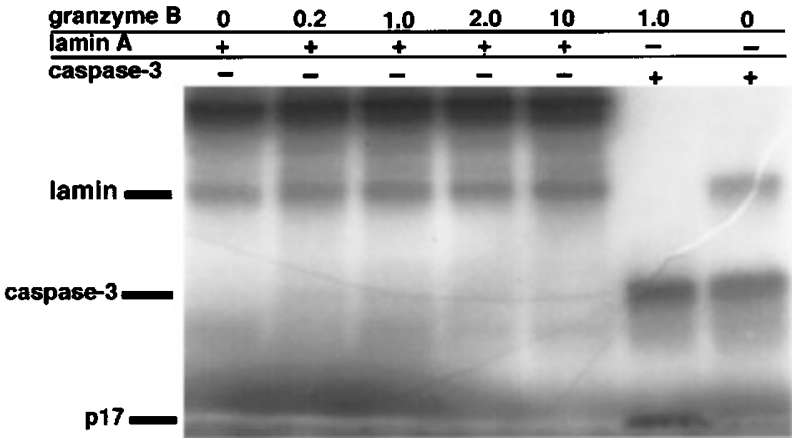

Figure 6 Granzyme B does not directly cleave lamin A. Radiolabeled Lamin A was produced by coupled in vitro transcription and translation. Caspase-3 was produced in the same manner to serve as a positive control. Translation products were incubated in the noted concentration of granzyme $B$ for $60 \mathrm{~min}$ at $37^{\circ} \mathrm{C}$ and run on a $10 \%$ polyacrylamide gel, fixed in enhancing solution and exposed to X-ray film

After Ad2 stimulation of transfected COS cells, granzyme $B$ positive cells displayed a high level of nuclear granzyme B and were essentially 100\% TUNEL positive (Figures 2 and 3). COS M5 cells expressing granzyme $\mathrm{B}[\mathrm{S} 183 \mathrm{~A}]$ also displayed Ad2 dependent nuclear labeling of granzyme B, but were not TUNEL positive (Figure $3 \mathrm{C}$ ). Analysis of CLSM micrographs showed there was a fivefold increase of granzyme $B$ in nuclei of cells treated with granzyme $\mathrm{B}[\mathrm{S} 183 \mathrm{~A}]$ active site mutant (Figure $2 B, D)$ compared with cells in the absence of any granzyme B treatment. It is important to note the increase in relative nuclear inactive granzyme $B$ in the absence of DNA fragmentation and TUNEL reactivity. However, the level of nuclear granzyme B[S183A] was lower than wildtype granzyme B. Results from these two approaches demonstrate that nuclear translocation of granzyme $B$ is 
not absolutely dependent on enzymatic activity, but is markedly increased when the active enzyme is present. However, the use of inactivated or mutant granzyme B resulted in the complete absence of the biochemical and morphological indicators of apoptosis.

\section{Caspase-3 is involved in granzyme B-mediated DNA fragmentation, but not nuclear condensation}

Granzyme B has been shown to cleave and activate caspase-3 in vitro ${ }^{25}$ and to be processed in targets exposed to either whole CTL or the combination of granzyme B and $A d 2.5,26$ To investigate the role of caspase-3 in the development of apoptotic morphology,

A.

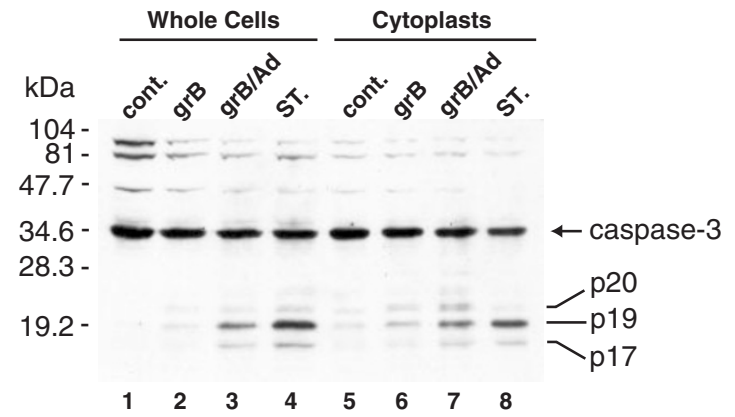

B.

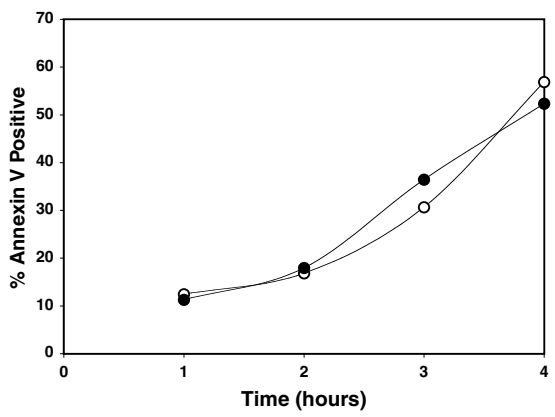

C.

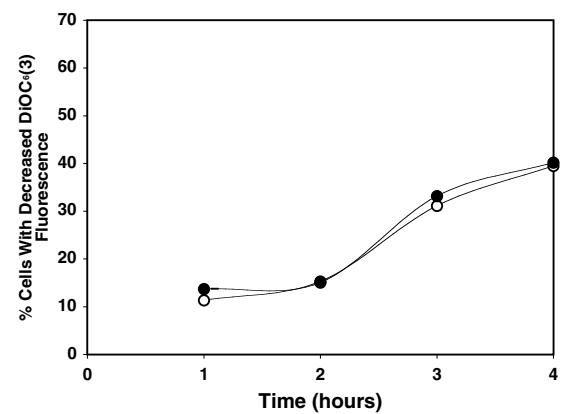

Figure 7 Cleavage of caspase-3 in granzyme B/Ad2-treated cells does not require a nucleus. Western blot of protein lysates from Jurkat cells and Jurkat cytoplasts treated with granzyme and Ad2, probed with an antibody recognizing the p17 subunit of pro-caspase-3 and caspase- 3 . The appearance of the p17 subunit of activated caspase- 3 is similar in both cytoplasts and in nucleated cells and is comparable between granzyme B/Ad2 and staurosporine-treated cells the effect of the inhibitor of caspase-3-like caspases, benzyloxycarbonyl-Asp-Glu-Val-Asp-fluoromethyl ketone (zDEVD-fmk), was evaluated in our model system. In the presence of $z$-DEVD-fmk, Jurkat and COS M5 targets treated with granzyme B and Ad2 failed to develop TUNEL reactivity (Figure 4), suggesting that the inhibitor prevented the form of DNA fragmentation recognized by this technique. Despite the absence of DNA fragmentation, immunolocalization studies demonstrate that the amount of granzyme B detected in the nucleus was drastically reduced when caspase-3-like caspases were inactivated with z-DEVD-fmk, suggesting that some caspase-3dependent event may facilitate translocation of granzyme into the nucleus (Figures 2 and 3 ).

To test the hypothesis that nuclear condensation was not mediated by caspase-3, we assessed nuclear morphology of target cells labeled with the DNA binding dye, DAPI. Cells that were treated with $z$-DEVD-fmk followed by granzyme B and Ad2 or perforin are shown in Figure 5. Although these cells were not TUNEL positive (Figure 4), many showed signs of condensed nuclei. (Figure 5). These results demonstrate that nuclear condensation in granzyme B-mediated apoptosis is mediated via some activity other than caspase-3. These data support the notion that granzyme B-dependent DNA fragmentation is mediated through activation of caspase-3 or another caspase and that the granzyme induces nuclear condensation indirectly, likely through the activation of the laminase, caspase-6.

Proteolytic degradation of the nuclear lamina is an integral step in the dismantling of an apoptotic cell. Although caspase- 6 (Mch2 $\alpha$ ) has recently been shown to be responsible for laminase activity in HeLa nuclei, there remained the possibility that cleavage of lamin A was due to granzyme B activity in granule-mediated killing of targets. To test the potential proteolytic activity of granzyme B toward lamin A, we used lamin A that was transcribed and translated in vitro from the full length open reading frame cDNA cloned into the expression vector Bluescript pSK+. ${ }^{35}$ S-Met lamin A was incubated for $30 \mathrm{~min}$ in the presence of granzyme B. Caspase-3, which was generated in the same manner to avoid the possibility of inhibitors being present in the translation mixture, was used as a positive control for granzyme B activity. Granzyme B, in amounts sufficient to cleave caspase-3, did not cleave lamin A. (Figure 6).

\section{Loss of membrane asymmetry is not dependent on nuclear translocation of granzyme B}

To test the involvement of nuclear granzyme B in cytoplasmic aspects of apoptosis, we performed granzyme B killing assays on enucleated target cells. Jurkat cells were treated with $2 \mu \mathrm{g} / \mathrm{ml}$ cytochalasin B for $30 \mathrm{~min}$ at $37^{\circ} \mathrm{C}$ and then centrifuged at $80000 \times g$ for 30 min over a discontinuous Percoll gradient to separate enucleated cytoplasts from intact cells and nuclei. Cytoplasts were treated as above with granzyme B and Ad2 for 30,60 and 120 min and apoptosis was assessed by the binding of annexin V-FITC to phosphatidylserine moieties displayed on the extracellular 
surface of the plasma membrane. Analysis by flow cytometry showed that there was no reduction in the amount or kinetics of phosphatidylserine flip observed in granzyme B/Ad2treated cytoplasts compared with intact targets that were isolated from a similar Percoll gradient in the absence of cytochalasin B (data not shown). This demonstrates that nuclear translocation of granzyme $B$ is not required for the exposure of phosphatidylserine on the extracellular face of the plasma membrane.

Additionally, we also assessed the changes in mitochondrial inner membrane potential, $\Delta \Psi_{\mathrm{m}}$, and the cleavage of caspase-3 in granzyme B/Ad2-treated Jurkat and Jurkat cytoplasts. We observed the appearance of the $\mathrm{p} 17$ subunit of processed caspase- 3 in both the nucleated and enucleated targets with similar kinetics (Figure 7). Further, staurosporine-treated Jurkat cells and cytoplasts displayed similar caspase-3 cleavage. We did not observe any reduction in the loss of $\Delta \Psi_{\mathrm{m}}$ in enucleated target cytoplasts as compared to $\Delta \Psi_{\mathrm{m}}$ loss in Jurkat cells (Figure 7C).

\section{Discussion}

In this report we demonstrate that granzyme B is rapidly translocated to the nucleus of target cells that are fated to undergo apoptosis. Earlier we demonstrated that granzyme B enters the target cell autonomously and resides in an intracellular vesicle unless perforin or Ad2 is added to the target. ${ }^{5}$ Once perforin is added, the appearance of nuclear granzyme B precedes the onset of DNA fragmentation. The mechanism that results in nuclear localization of granzyme $B$ is unclear. Granzyme B does not contain a linear or bipartite nuclear localization signal, ${ }^{27,28}$ nor is the granzyme involved in known processes of nuclear import. ${ }^{21}$ There remains a distinct possibility that translocation is facilitated by a carrier protein. $^{28,29}$

Activation of caspase-3 by granzyme $B$ has been shown to be required for granule-mediated apoptosis. ${ }^{25}$ To investigate the involvement of caspase-3, we used an irreversible substrate analog inhibitor, z-DEVD-fmk, which inhibits caspase-3-like caspases. The localization of caspase-3 in the cytosol and the necessity of caspase-3 to produce DNA fragmentation suggests that granzyme $B$ cleaves caspase-3 in the cytoplasm prior to nuclear translocation of both proteinases, where caspase- 3 is responsible for cleaving poly(ADP-ribose) polymerase (PARP) and other nuclear substrates. ${ }^{30,31}$ Consistent with published reports of the localization of caspase- $3,{ }^{32,33}$ we observed a cytoplasmic labeling pattern for caspase-3 in Jurkat cells that were not undergoing apoptosis (data not shown). Since granzyme B does not require a target cell nucleus for cleavage of caspase-3, then presumably this event is prior to granzyme B being translocated to the nucleus. Also, given that translocation of granzyme $B$ into the nucleus is diminished in the presence of the caspase-3 inhibitor, z-DEVD-fmk, it is possible that some caspase-3mediated event is prior to translocation of granzyme $B$. However, it is also possible that there is some other inhibitory effect(s) of z-DEVD-fmk, but it is not likely to be as a result of inhibition of known caspases (notably caspase-8) since granzyme B has been clearly demonstrated to cleave caspase-3 and other effector caspases directly. $25,26,34,35$

$\mathrm{DCl}$-inactivated granzyme B was observed inside the targets and, after addition of perforin or Ad2, was translocated to the nucleus, but at reduced levels. DNA fragmentation, however, was not observed in these cells. It is unlikely that this is due to incomplete inactivation of the proteolytic activity as perforin and Ad2 both stimulated nuclear translocation of catalytically inactive granzyme $\mathrm{B}[\mathrm{S} 183 \mathrm{~A}]$ in COS cells without DNA fragmentation. However, when catalytically active protease was used in these two models for granzyme B delivery, DNA fragmentation was always observed. From these results we concluded that enzymatic activity of granzyme B, although not required for binding and internalization, ${ }^{5}$ facilitates efficient translocation to the nucleus and is essential for cell death. Given that we observed a reduced, but significant translocation of enzymatically inactive granzyme $B$, we cannot rule out the possibility of a granzyme Bindependent activation of caspase-3, or similar caspase, that facilitated a limited nuclear translocation of granzyme. Interestingly, it has recently been shown that catalytically inactive granzyme $A$ still retains the ability to induce membrane damage in target cells. ${ }^{36}$

When cells were treated with z-DEVD-fmk there was an abrogation of DNA fragmentation. Strikingly, nuclear condensation was still observed in these cells. This suggests that, although granzyme B-mediated DNA fragmentation is dependent upon caspase-3 activation, granzyme $B$ acts on nuclear substrates to cause nuclear condensation. ${ }^{37}$

Lamin proteolysis is believed to be one of the key steps in dismantling of the apoptotic nucleus. Since we had observed nuclear condensation in the absence of DNA fragmentation, it was apparent that lamin proteolysis was occurring in the absence of active caspase-3. We used in vitro transcribed and translated lamin $A$ to test lamin $A$ as a possible substrate for granzyme B. With amounts of granzyme sufficient to cleave caspase-3, we observed no cleavage of lamin A. Since granzyme B did not cleave lamin $A$ in vitro, it is likely that granzyme $B$ triggers nuclear condensation through the activation of other proteases that are not inhibited by the caspase-3 inhibitor, z-DEVD-fmk.

By using enucleated target cells we have addressed the question of the possible involvement of nuclear granzyme B in the cytoplasmic events commonly observed in apoptosis. Since the nucleus was not necessary for granzyme $B$ to induce phosphatidylserine flip, loss of $\Delta \Psi_{\mathrm{m}}$ or caspase-3 cleavage, we conclude that the relevance of nuclear granzyme B is restricted to events required for apoptotic degradation of the target cell nucleus. It is important to note that although the target cell nucleus is dispensable for the cytoplasmic events in granzyme B-mediated apoptosis, the importance of the nuclear events should not be discounted, since the nucleus is a major component of physiological targets of cytotoxic $\mathrm{T}$ cells. The possibility of microinjecting granzyme B directly into the nucleus may provide us with further insights into the role of nuclear granzyme B. 
Jans et $a l^{38}$ demonstrated that perforin is required for granzyme $B$ to translocate to the nucleus in cells that were mechanically disrupted to permit the movement of granzyme. In a previous report, we established that granzyme B recognizes a nuclear protein associated with heterochromatin. ${ }^{7}$ Here we add further evidence to suggest a nuclear role for granzyme $\mathrm{B}$, by demonstrating that granzyme $\mathrm{B}$ is rapidly translocated to the nucleus upon induction of apoptosis in vivo. Nuclear translocation of granzyme is not absolutely dependent on enzymatic activity but is enhanced when the active protease is present. We also show that DNA fragmentation, which follows the appearance of nuclear granzyme B, is dependent on active granzyme and is mediated through activation of casapse-3, whereas nuclear condensation mediated by granzyme B occurs independently of caspase-3.

\section{Materials and Methods}

\section{Cells and reagents}

Jurkat and COS M5 cells were cultured as previously described. ${ }^{5}$ Jurkat cytoplasts were produced by treatment of Jurkat in $2 \mu \mathrm{g} / \mathrm{ml}$ cytochalasin $\mathrm{B}$ (Sigma) for $30 \mathrm{~min}$ at $37^{\circ} \mathrm{C}$ followed by centrifugation over a Percoll gradient of 25,16 and $12 \%$ for $30 \mathrm{~min}$ at $80000 \times \mathrm{g}$. The cytoplasts were collected from the top (least dense) layer and washed three times in media to remove Percoll and cytochalasin B. As a control for the treatment, Jurkat that were not treated with cytochalasin B were also run over a Percoll gradient and were collected from the interface between the 12 and $16 \%$ Percoll.

Granzyme B constructs in the vector pAX142 were transiently transfected into COS M5 fibroblasts by standard DEAE-dextran protocol as previously described. ${ }^{38}$ Enzymatic activity of mature granzyme $B$ was assayed by cleavage of the synthetic substrate tertbutyloxycarbonyl-Ala-Ala-Asp-thiobenzylester in the presence of the chromogenic indicator dithiobis(2-nitrobenzoic acid) as described. ${ }^{39}$

Antisera against residues $9-16$ of granzyme $B$, provided by Dorothy Hudig (University of Nevada, Reno, NV, USA) was generated as previously described. ${ }^{7}$ Goat anti-rabbit-FITC, goat anti-rabbitTexas Red ${ }^{\mathrm{TM}}$, goat anti-mouse Texas Red ${ }^{\mathrm{TM}}$ and mouse anti-rabbit rhodamine secondary antibodies were purchased from Jackson Immunoresearch. Rabbit polyclonal antisera recognizing the p17 subunit of caspase-3 was provided by Don Nicholson (Merck Frosst, Kirkland, Quebec, Canada).

Perforin and granzyme B were purified from the human cytolytic cell line YT, as previously described. ${ }^{40}$ Granzyme B and Type 2 adenovirus (Ad2), purified according to Seth et al. ${ }^{41}$ was kindly provided by Dr. Chris Froelich, Northwestern University, Evanston, Illinois. Wild-type murine granzyme $B$ and an enzymatically inactive mutant granzyme $\mathrm{B}[\mathrm{S} 183 \mathrm{~A}]$ were expressed using the pAX142 expression vector by transient transfection of COS-M5 cells as described previously. ${ }^{39}$

\section{Induction of apoptosis}

Granzyme B was added directly to Jurkat target cells at $1 \mu \mathrm{g} / \mathrm{ml}$ in RPMI supplemented with BSA $(0.05 \% \mathrm{w} / \mathrm{v})$. Sublytic doses of perforin were used at $90 \mathrm{U} / \mathrm{ml}$, where one unit is defined in the standard sheep red blood cell hemolytic assay. Cells were washed in media prior to further treatment. ${ }^{5}$ Volumes equivalent to the incubation volume were used for each wash. Infection with Ad2 was performed in RPMI with
BSA $(0.05 \%)$, as noted above for granzyme B, at a multiplicity of infection of ten plaque forming units per cell. Transiently transfected COS M5 cells were treated with Ad2 or perforin, as described above, without prior treatment of granzyme B. Inhibition studies with caspase3 inhibitor benzyloxycarbonyl-Asp-Glu-Val-Asp-fluoromethyl ketone (z-DEVD-fmk) (Kamiya, Seattle, WA, USA) were performed by incubating target cells in the presence of the inhibitor $(20 \mu \mathrm{M})$ for $30 \mathrm{~min}$ prior to addition of perforin or Ad2. All cells were incubated at $37^{\circ} \mathrm{C}$ unless otherwise noted.

\section{Assessment of apoptosis}

Nuclear morphology was assessed by staining with the DNA-binding dye, 4,6-diamidino-2-phenylindole-dihydrochloride (DAPI). Cells were permeabilized for $15 \mathrm{~min}$ in $0.1 \%$ saponin in PBS prior to labeling and incubated for $15 \mathrm{~min}$ in DAPI at $1 \mu \mathrm{g} / \mathrm{ml}$ in PBS/saponin. Visualization of DAPI-stained cells was performed with a Zeiss fluorescence microscope. Phosphatidylserine externalization from the inner to the outer leaflet of the plasma membrane was assayed by measurement of annexin V-FITC binding (Clontech) to live cells in bulk culture by flow cytometry with a Becton-Dickinson FACSCAN. Cells were analyzed within $15 \mathrm{~min}$ following the addition of annexin V-FITC. DNA fragmentation was assessed in situ by the TUNEL method ${ }^{42}$ using terminal transferase and dUTP-FITC (Boehringer Mannheim Corp.) in saponin-permeabilized cells. TUNEL-labeled cells were assayed by fluorescent microscopy as described in detail previously. ${ }^{5}$ The inner mitochondrial membrane potential $\left(\Delta \Psi_{m}\right)$ was measured using $3,3^{\prime}$ dihexyloxacarbocyanine iodide $\left(\mathrm{DiOC}_{6}(3)\right)$ as previously described. ${ }^{43}$ Detection of caspase- 3 cleavage was determined by Western blotting as described previously. ${ }^{35}$

Jurkat cells were attached directly to glass microscope slides by centrifugation for $15 \mathrm{~s}$ at $600 \times \mathrm{g}$. COS M5 cells were grown directly onto $0.5 \mathrm{~mm}$ glass coverslips. All cells were fixed immediately in paraformaldehyde ( $2 \% \mathrm{w} / \mathrm{v}$ in PBS) and washed in PBS prior to permeabilization and immunolabeling. Cells were labeled as previously described ${ }^{5}$ and viewed with a Zeiss fluorescence microscope or by confocal laser scanning microscopy and analyzed with the accompanying CLSM software (CLSM; Leica, Heidelberg, Germany). Images were acquired by 32- or 64-line scan averaging using $100 \times / 1.32$ N.A. objective under oil immersion.

Adherent cells were grown directly onto glass cover slips and cells from suspension cultures were affixed to glass microscope slides by centrifugation for $15-20 \mathrm{~s}$ at $600 \times g$ in a Cytospin centrifuge. Cells were fixed in either $1 \%$ formalyn or $2 \%$ paraformaldehyde for $15 \mathrm{~min}$, washed in PBS for 15 min before labeling.

For immunolabeling, cells were permeabilized in saponin $(0.1 \%$ $\mathrm{w} / \mathrm{v}$ in PBS) for $15 \mathrm{~min}$ and blocked in non-fat milk powder (3\% w/v in PBS-saponin) for $30 \mathrm{~min}$ at room temperature (RT). Primary antigranzyme antiserum was added in blocking solution at a dilution of $1: 100$ and incubated for $30-60 \mathrm{~min}$ at RT, washed three times in PBS-saponin and incubated in fluorophore-conjugated secondary antibody for 30-60 min at RT. Cells were washed three times in PBSsaponin, dried, mounted and assessed visually within $48 \mathrm{~h}$.

\section{Quantification of CLSM images}

Digital images acquired on the confocal laser-scanning microscope were analyzed with the $\mathrm{NIH}$-Image Software to determine the relative level of label corresponding to granzyme B in the cytoplasm and nucleus in TUNEL positive and negative cells. Black and white images were sharpened, edged and thresholded. The Grain Counting Macro was used to count pixels corresponding to immunolabel. 


\section{Acknowledgements}

MJ Pinkoski is the recipient of a Post-Doctoral Fellowship from the Medical Research Council (MRC) of Canada. JA Heilbein holds an MRC Studentship, M Barry is the recipient of a Post-Doctoral Fellowship from the Alberta Heritage Medical Foundation for Medical Research (AHFMR), and RC Bleackley is a Medical Scientist of the AHFMR, an MRC Distinguished Scientist and an International Scholar of the Howard Hughes Medical Institute. We thank Don Nicholson for the anti-caspase-3 antibody, Chris Froelich for purified granzyme B, and Prem Seth for the replication-deficient adenovirus.

\section{References}

1. Berke $G$ (1994) The binding and lysis of target cells by cytotoxic lymphocytes: molecular and cellular aspects. Annu. Rev. Immunol. 12: 735-773

2. Berke G (1995) The CTL's kiss of death. Cell $81: 9-12$

3. Darmon AJ and Bleackley RC (1998) Proteases and cell-mediated cytotoxicity. Crit. Rev. Immunol. 18: 255-273

4. Darmon AJ, Pinkoski MJ and Bleackley RC (1999) Granule-mediated cytotoxicity. Results Probl. Cell Differ. 23: 103-125

5. Pinkoski MJ, Hobman M, Heibein JA, Tomaselli K, Li F, Seth P, Froelich CJ and Bleackley RC (1998) Entry and trafficking of granzyme B in target cells during granzyme B- perforin-mediated apoptosis. Blood 92: 1044-1054

6. Shi L, Mai S, Israels S, Browne K, Trapani JA and Greenberg AH (1997) Granzyme B (GraB) autonomously crosses the cell membrane and perforin initiates apoptosis and GraB nuclear localization. J Exp Med 185: 855-866

7. Pinkoski MJ, WinklerU, Hudig D and Bleackley RC (1996)Binding of granzyme B in the nucleus of target cells. Recognition of an 80-kilodalton protein. J Biol Chem 271: $10225-10229$

8. Trapani JA, Browne KA, Smyth MJ and Jans DA (1996) Localization of granzyme $B$ in the nucleus. A putative role in the mechanism of cytotoxic lymphocyte-mediated apoptosis. J. Biol. Chem. 271: 4127-4133

9. Trapani JA, Jans P, Smyth MJ, Froelich CJ, Williams EA, Sutton VRand Jans DA (1998) Perforin-dependent nuclear entry of granzyme B precedes apoptosis, and is not a consequence of nuclear membrane dysfunction. Cell Death Differ. 5 488-496

10. Trapani JA, Jans DA, Jans PJ, Smyth MJ, Browne KA and Sutton VR (1998) Efficient nuclear targeting of granzyme $B$ and the nuclear consequences of apoptosis induced by granzyme $B$ and perforin are caspase-dependent, but cel death is caspase-independent. J Biol Chem 273: 27934-27938

11. Sellins KS and Cohen JJ (1995) Nuclear changes in the cytotoxic T lymphocyteinduced model of apoptosis. Immunol. Rev. 146: 241-266

12. Wyllie AH (1980) Glucocorticoid-induced thymocyte apoptosis is associated with endogenous endonuclease activation. Nature 284: 555-556.

13. Wyllie AH, Morris RG, Smith AL and Dunlop D (1984) Chromatin cleavage in apoptosis: association with condensed chromatin morphology and dependence on macromolecular synthesis. J. Pathol. 142: 67-77

14. Duke RC, Chervenak R and Cohen JJ (1983) Endogenous endonucleaseinduced DNA fragmentation: an early eventin cell-mediated cytolysis. Proc. Natl. Acad. Sci. USA 80: 6361-6365

15. McKeon F (1991) Nuclearlamin proteins: domains required for nuclear targeting, assembly, and cell-cycle-regulated dynamics. Curr. Opin. Cell Biol. 3: 82-86

16. Lazebnik YA, Takahashi A, Moir RD, Goldman RD, Poirier GG, Kaufmann SH and Earnshaw WC (1995) Studies of the lamin proteinase reveal multiple paralle biochemical pathways during apoptotic execution. Proc. Natl. Acad. Sci. USA 92: 9042-9046

17. Takahashi A, Alnemri ES, Lazebnik YA, Fernandes-Alnemri T, Litwack G, Moir RD, Goldman RD, Poirier GG, Kaufmann SHand Earnshaw WC (1996)Cleavage of lamin A by Mch2 alpha but not CPP32: multiple interleukin 1 beta-converting enzyme-related proteases with distinct substrate recognition properties are active in apoptosis. Proc. Natl. Acad. Sci. USA 93: 8395-8400

18. Sakahira H, Enari M and Nagata $S$ (1998) Cleavage of CAD inhibitor in $C A D$ activation and DNA degradation during apoptosis. Nature 391: $96-99$

19. Liu X, Zou H, Slaughter $C$ and Wang X (1997) DFF, a heterodimeric protein that functions downstream of caspase-3 to trigger DNA fragmentation during apoptosis. Cell 89: 175-184
20. Enari M, Sakahira H, Yokoyama H, Okawa K, Iwamatsu A and Nagata S (1998) A caspase-activated DNase that degrades DNA during apoptosis, and its inhibitor ICAD. Nature 391: 43-50

21. Jans DA and Hubner S (1996) Regulation of protein transport to the nucleus: central role of phosphorylation. Physiol. Rev. 76: 651-685

22. Jans DA, Sutton VR, Jans P, Froelich CJ and Trapani JA (1999) BCL-2 blocks perforin-induced nuclear translocation of granzymes concomitant with protection against the nuclear events of apoptosis. J Biol Chem 274: 3953-3961

23. Jans DA, Briggs LJ, Jans $P$, Froelich CJ, Parasivam G, Kumar S, Sutton VR and Trapani JA (1998) Nuclear targeting of the serine protease granzyme A (fragmentin-1). J. Cell Sci. 111: 2645-2654

24. Hudig D, Allison NJ, Pickett TM, Winkler U, Kam CM and Powers JC (1991) The function of lymphocyte proteases. Inhibition and restoration of granule-mediated Iysis with isocoumarin serine protease inhibitors. J. Immunol. 147: 1360-1368

25. Darmon AJ, Nicholson DW and Bleackley RC (1995) Activation of the apoptotic protease CPP32 by cytotoxic T-cell-derived granzyme B. Nature 377: 446-448

26. Darmon AJ, Ley TJ, Nicholson DW and Bleackley RC (1996) Cleavage of CPP32 by granzyme $B$ represents a critical role for granzyme $B$ in the induction of target cell DNA fragmentation. J. Biol. Chem. 271:21709-21712

27. Gorlich D and Mattaj IW (1996) Nucleocytoplasmic transport. Science 271: $1513-1518$

28. Davis LI (1995) The nuclear pore complex. Annu. Rev. Biochem. 64: 865-896

29. Nigg EA (1997) Nucleocytoplasmic transport: signals, mechanisms and regulation. Nature 386: $779-787$

30. Nicholson DW, Ali A, Thornberry NA, Vaillancourt JP, Ding CK, Gallant M, Gareau Y, Griffin PR, Labelle M and Lazebnik YA, et al. (1995) Identification and inhibition of the ICE/CED-3 protease necessary for mammalian apoptosis. Nature 376: 37-43

31. Tewari M, Quan LT, O'Rourke K, Desnoyers S, Zeng Z, Beidler DR, Poirier GG, Salvesen GS and Dixit VM (1995) Yama/CPP32 beta, a mammalian homolog of CED-3, is a CrmA-inhibitable protease that cleaves the death substrate poly(ADP-ribose) polymerase. Cell 81: 801-809

32. Samali A, Zhivotovsky B, Jones DP and Orrenius S (1998) Detection of pro-caspase-3 in cytosol and mitochondria of various tissues. FEBS Lett. 431: 167-169

33. Chandler JM, Cohen GM and MacFarlane M (1998) Different subcellular distribution of caspase-3 and caspase-7 following Fas-induced apoptosis in mouse liver. J. Biol. Chem. 273: 10815-10818

34. Chinnaiyan AM, Hanna WL, Orth K, Duan H, Poirier GG, Froelich CJ and Dixit VM (1996) Cytotoxic T-cell-derived granzyme B activates the apoptotic protease ICE-LAP3. Curr. Biol. 6: 897-899

35. Atkinson EA, Barry M, Darmon AJ, Shostak I, Turner PC, Moyer RW and Bleackley RC (1998) Cytotoxic T lymphocyte-assisted suicide. Caspase 3 activation is primarily the result of the direct action of granzyme B. J. Biol. Chem. 273: $21261-21266$

36. Beresford PJ, Zhinan X, Greenberg AH and Lieberman J (1999) Granzyme A loading induces rapid cytolysis and a novel form of DNA damage independently of caspase activation. Immunity 10: 585-594

37. Andrande F, Roy S, Nicholson D, Thornberry N, Rosen A and Casciola-Rosen L (1998) Granzyme B directly and efficiently cleaves several downstream caspase substrates: implications for CTL-induced apoptosis. Immunity 8: $451-460$

38. Jans DA, Jans P, Briggs LJ, Sutton V and Trapani JA (1996) Nuclear transport of granzyme $B$ (fragmentin-2). Dependence of perforin in vivo and cytosolic factors in vitro. J. Biol. Chem. 271: 30781-30789

39. Caputo A, Garner RS, Winkler U, Hudig D and Bleackley RC (1993) Activation of recombinant murine cytotoxic cell proteinase- 1 requires deletion of an aminoterminal dipeptide. J. Biol. Chem. 268: 17672-17675

40. Hanna WL, Zhang X, Turbov J, Winkler U, Hudig D and Froelich CJ (1993) Rapid purification of cationic granule proteases: application to human granzymes. Protein Expr. Purif. 4: 398-404

41. Seth $P$, Fitzgerald D, Willingham M and Pastan I (1986) Pathway of adenovirus entry into cells. In: Virus Attachment and Entry into Cells. Washington, D.C.: American Society for Microbiology; pp. 191-195

42. Gavrieli $Y$, Sherman $Y$ and Ben-Sasson SA (1992) Identification of programmed cell death in situ via specific labeling of nuclear DNA fragmentation. J. Cell. Biol. 119: 493-501

43. Heibein JA, Barry M, Motyka B and Bleackley RC (1999) Granzyme B-induced loss of mitochondrial inner membrane potential $\left(\Delta \Psi_{\mathrm{m}}\right)$ and cytochrome c release are caspase-independent. J. Immunol, 163: 4683-4693 